\section{Changing Landscape for Canadian Public}

\section{Health}

My second editor's page starts as did the first one with a thank you, or more appropriately, "merci"! "Merci beaucoup" in fact is in order for the two associate editors who have managed for six years the French language submissions to the Journal, Drs Denise Aubé and Clément Beaucage. We are deeply grateful for their excellent work and for their contributions to the quality of French manuscripts we have published. Two new interim associate editors will take over the management of French manuscripts in the fall: Dr Benoît Lévesque and Mrs Claire Laliberté, from the Institut national de santé publique du Québec (INSPQ). Their expertise and experience in environmental health will be a valuable addition to the Journal.

Change seems to be the order of the day - and of the past few years - for public health in Canada. Following the SARS, Walkerton and BSE crises and the subsequent reports which raised attention about the sorry state of our public health system, the landscape has changed quite dramatically. Several of these changes were at least in part due to the dynamic presence of CIHR's Institute of Population and Public Health, which not only defined innovative perspectives about health research in Canada but also helped catalyze the forces of public health across the country in support of a renewed and modern system.

The creation of the Public Health Agency of Canada (PHAC) was a bold (and long overdue) move on the part of the federal government which could, over time, drastically alter the practice of public health in Canada. The Agency has much on its plate but it has already taken steps to support the training of new public health professionals and researchers and to support local public health organizations. Provincial counterparts to PHAC are also in the works in some provinces, including Quebec's INSPQ which antedates PHAC by several years, to consolidate and reinforce provincial expertise. The Canadian Population Health Initiative has also contributed to our improved understanding of the determinants of health and the dissemination of this evidence to Canadians. Finally, CPHA has also seen major changes and modernization of its governance model over the past few years. Amid all these changes, we must not overlook the capacity development needs at the local level. In addition, the activities of these organizations will have to be coordinated to maximize the health benefits to the Canadian population. This will not be the least of their problems.

Gilles Paradis, MD, MSc, FRCPC, FACPM, FAHA

Interim Scientific Editor, CJPH
LE MOT DE LA RÉDACTION

\section{La santé publique au Canada : un paysage en transformation}

Mon deuxième éditorial commence, comme le premier, par un remerciement. Merci, merci beaucoup, aux docteurs Denise Aubé et Clément Beaucage, les deux rédacteurs adjoints qui se sont occupés, pendant les six dernières années, des manuscrits français proposés à la Revue. Nous leur sommes infiniment reconnaissants pour leur excellent travail et pour leur apport à la qualité des articles publiés en français. Deux nouveaux rédacteurs adjoints prendront en charge les manuscrits français cet automne : le $\mathrm{D}^{\mathrm{r}}$ Benoît Lévesque et $\mathrm{M}^{\mathrm{me}}$ Claire Laliberté, tous deux de l'Institut national de santé publique du Québec (INSPQ). Leurs compétences et leur expérience dans le domaine de la salubrité de l'environnement seront un atout de plus pour la Revue.

Dans les milieux de la santé publique au Canada, le changement est au menu du jour... depuis plusieurs années. Les crises du SRAS, de Walkerton et de l'ESB et les rapports qui ont suivi ont permis de constater l'état déplorable du réseau de la santé publique. Depuis, le paysage institutionnel s'est profondément transformé. Plusieurs de ces transformations se sont opérées, au moins en partie, grâce à la présence dynamique de l'Institut de la santé publique et des populations des IRSC, lequel a non seulement ouvert des perspectives novatrices pour la recherche en santé au Canada, mais a aidé à catalyser les forces vives de la santé publique du pays à l'appui d'un renouvellement et d'une modernisation du réseau.

La création de l'Agence de santé publique du Canada fut un geste audacieux (même s'il s'imposait depuis longtemps) de la part du gouvernement fédéral. Avec le temps, cette agence pourrait radicalement changer la pratique de la santé publique au pays. L'Agence a beaucoup de pain sur la planche, mais elle prend déjà des mesures pour appuyer la formation des jeunes professionnels et chercheurs et pour soutenir les organismes locaux en santé publique. Des organismes de contrepartie existent déjà ou sont en projet dans plusieurs provinces, notamment au Québec, où la création de l'INSPQ a précédé de plusieurs années celle de l'Agence fédérale, contribuant ainsi à regrouper et à renforcer le savoir dans la province. Par ailleurs, l'Initiative sur la santé de la population canadienne a amélioré nos connaissances des déterminants de la santé et la diffusion de ces connaissances dans la population canadienne. Et l'Association canadienne de santé publique a entièrement remanié et modernisé son modèle de gouvernance ces dernières années. Tous ces changements ne doivent pas nous faire oublier qu'il faut aussi renforcer les capacités locales en santé publique. De plus, les organismes mentionnés devront coordonner leur action de manière à en maximiser les avantages pour la santé de la population canadienne. La tâche sera loin d'être facile.

Le rédacteur-réviseur scientifique intérimaire de la $R C S P$,

Gilles Paradis, M.D., M.Sc., FRCPC, FACPM, FAHA 